SCIENTIFIC LETTER

\title{
Impact of asynchronous ventricular activation on pro- inflammatory cytokines and oxidative stress in paced patients
}

\author{
M E Marketou, E N Simantirakis, D Nikitovic, S I Chrysostomakis, E A Zacharis, P E Vardas
}

Heart 2005;91:817-818. doi: 10.1136/hrt.2004.039925

A synchronous ventricular activation (AVA), caused by right apical ventricular pacing (RAVP), results in the deterioration of both systolic and diastolic function. ${ }^{12}$ Chronic RAVP under DDDR pacing mode in patients with sick sinus syndrome revealed a detrimental effect on left ventricular fractional shortening, left atrium dilation, and myocardial blood flow. ${ }^{4}$

Furthermore, although the mechanism has not been fully elucidated, pro-inflammatory cytokines and oxidative stress have been shown, in both experimental and clinical studies, to affect the myocardium, compromising ventricular systolic performance. ${ }^{5}$

We hypothesised that these two latter factors are implicated in the functional and metabolic abnormalities in patients with AVA caused by RAVP. With this in mind we studied the concentrations of interleukin-6 (IL-6), tumour necrosis factor $\alpha(\mathrm{TNF} \alpha)$, and lipid peroxides (LP) in patients with sick sinus syndrome and a permanent dual chamber pacemaker during physiological and RAVP, as produced by AAIR and DDDR permanent pacing, respectively.

\section{METHODS}

We enrolled consecutive patients with sick sinus syndrome, first degree atrioventricular block (PR interval $\geqslant 200 \mathrm{~ms}$ ), and normal intraventricular conduction, who required a dual chamber pacemaker implantation.

The pacemaker was initially programmed as AAIR with a basic rate of $40 \mathrm{bpm}$ for a three month period and then the patients were randomised for 15 days to either AAIR or DDDR pacing, with a basic rate of $60 \mathrm{bpm}$, and the study continued in a crossover design. During DDDR pacing, the ventricular activation was fully paced and the atrioventricular interval was the one that ensured the best diastolic filling. At the end of each 15 day randomisation, but not at baseline, blood samples were taken to evaluate LP, IL-6, and TNF $\alpha$. An echocardiographic study was repeated at the end of every study period. All biomarkers were also measured in an acute crossover study involving one hour each of AAIR and DDDR pacing in random order.

Patients with a history of smoking, alcohol abuse, diabetes mellitus, hypertension, supraventricular arrhythmias, organic heart disease, or inflammatory or malignant disease were excluded. None of the patients were taking any medication that might have affected biomarker concentrations.

All the participants gave written informed consent. The study was approved by the ethical committee of the hospital.

Serum concentrations of TNF $\alpha$ (detection limit $0.18 \mathrm{pg} / \mathrm{ml}$ ) and IL-6 (detection limit $0.1 \mathrm{mg} / \mathrm{ml}$ ) were determined by a high sensitivity quantitative sandwich ELISA according to the instructions of the manufacturer (R\&D Systems, Abingdon, UK).
LP plasma concentration was measured by a photometric assay with a detection limit of $7 \mu \mathrm{mol} / \mathrm{l}$ (OxyStat, Biomedica, Vienna, Austria).

Data are summarised as mean (SD). Differences in LP, IL6 , and TNF $\alpha$ were assessed by repeated measures analysis of variance (ANOVA) with mode as the within factor at two levels (DDDR and AAIR) and order of mode pacing as the between factor. Interaction effects were also considered. Bonferroni adjusted 95\% confidence intervals (CI) were constructed to obtain a clearer picture of the size of the differences. The criterion for significance was $\mathrm{p}<0.05$.

\section{RESULTS}

A total of 25 patients ( 15 male; mean age 56 (8) years) were included. Ejection fraction, left atrial dimension, and wall motion indices were normal throughout the study and showed no significant changes. Anterior systolic interventricular septal motion was observed in 22 patients during DDDR pacing and in no patient during AAIR.

The one hour AAIR and DDDR pacing failed to show any significant acute effect (LP 348.7 (110.2) $\mu \mathrm{mol} / \mathrm{l}$, TNF $\alpha 3.2$ $(0.9) \mathrm{pg} / \mathrm{ml}, \quad$ IL-6 $2.6(0.8) \mathrm{pg} / \mathrm{ml}$ in AAIR $v \quad 351.2$ (102.6) $\mu \mathrm{mol} / \mathrm{l}, 3.1 \quad(0.9) \mathrm{pg} / \mathrm{ml}, 2.7(0.8) \mathrm{pg} / \mathrm{ml}$ in DDDR, $\mathrm{p}=0.73, \mathrm{p}=0.21, \mathrm{p}=0.58$, respectively).

In contrast to the acute study, LP and IL-6 concentrations were significantly elevated following the 15 day DDDR pacing period compared to those at the end of the AAIR pacing period (LP $338.3 \quad(116.6) \mu \mathrm{mol} / \mathrm{l}$ in AAIR $v 375.6$ (128.4) $\mu \mathrm{mol} / \mathrm{l}$ in DDDR, 95\% confidence interval (CI) of the difference from 17.83 to $56.88, \mathrm{p}=0.001$; and IL-6: 2.97 $(0.94) \mathrm{pg} / \mathrm{ml}$ in AAIR $v 3.21(0.95) \mathrm{pg} / \mathrm{ml}$, respectively, in DDDR, 95\% CI of the difference from 0.075 to 0.41 , $\mathrm{p}=0.006)$. TNF $\alpha$ tended to reveal a small but insignificant reduction during AAIR (3.25 (0.9) pg/ml in AAIR $v 3.48$ (1.07) $\mathrm{pg} / \mathrm{ml}$ in DDDR, $95 \% \mathrm{CI}$ of the difference from -0.014 to $0.47, \mathrm{p}=0.064$ ).

LP and IL-6 concentrations were significantly lower during AAIR pacing (fig 1) regardless of which phase came first (order effect: $95 \%$ CI of the difference from -26.22 to 168.04 , $\mathrm{p}=0.14$ for $\mathrm{LP}$, and $95 \% \mathrm{CI}$ of the difference from -0.752 to $0.825, \mathrm{p}=0.92$ for $(\mathrm{L}-6)$.

\section{DISCUSSION}

Our study demonstrates for the first time that AVA caused by RVAP in patients with sick sinus syndrome leads to an undesirable increase in pro-inflammatory cytokines and LP.

Abbreviations: AVA, asynchronous ventricular activation; IL-6, interleukin-6; LP, lipid peroxides; RAVP, right apical ventricular pacing; TNF $\alpha$, tumour necrosis factor $\alpha$ 

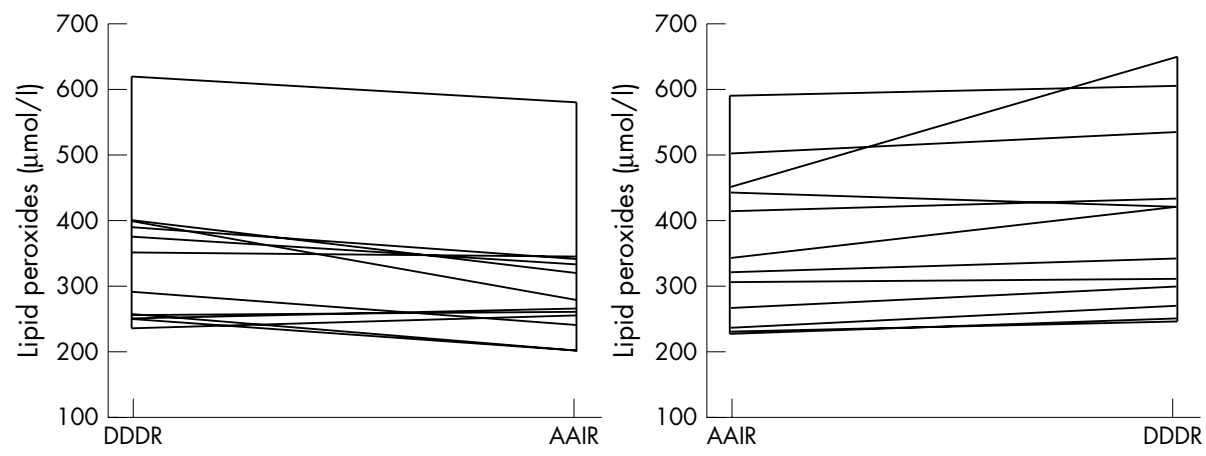

Figure 1 Lipid peroxide (upper panels) and interleukin-6 (lower panels) plasma concentrations were significantly lower during AAIR, regardless of which pacing mode was applied first.
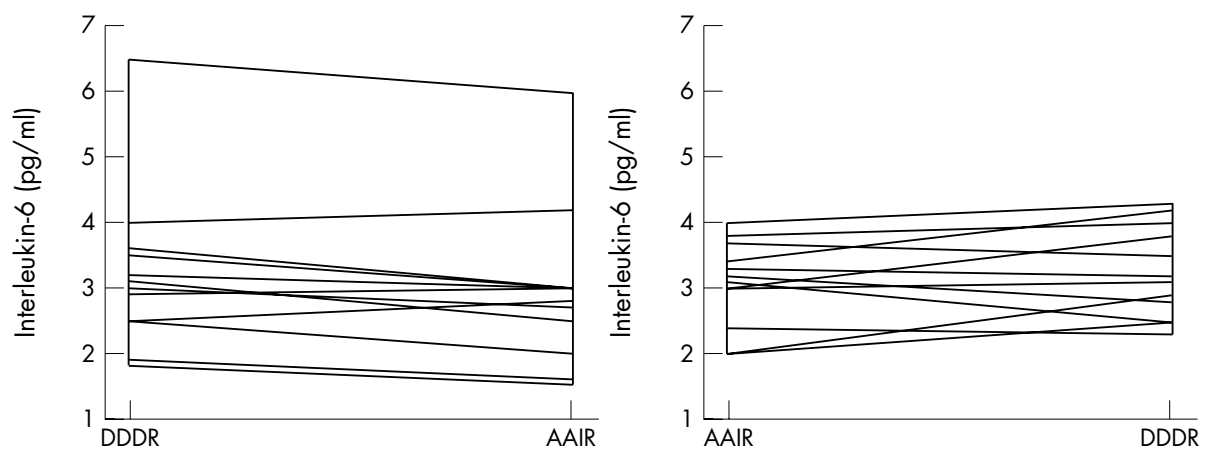

Published studies demonstrate that preserving the normal ventricular activation sequence acutely improves the cardiac systolic and diastolic performances and myocardial blood flow. ${ }^{124}$ On the other hand, it has been postulated that proinflammatory cytokines and oxidative stress are increased during ventricular dysfunction. ${ }^{5}$ Thus, the haemodynamically advantageous AAIR pacing may prevent the deleterious production of increased IL- 6 and LP. Our results could have even more serious implications for patients with myocardial ischaemia or heart failure. The non-significant increase of TNF $\alpha$ may be because of the short study period and DDDR permanent pacing in the long term might lead to an increase of $\mathrm{TNF} \alpha$ plasma concentrations in a similar manner.

We had no data concerning the baseline values for plasma concentrations of cytokines and LP. However, our aim was only to compare these two modes of pacing. In any case, no certain conclusions could have been drawn, since our data could have been influenced by differences in heart rate before and after the initiation of pacing.

Irrespective of the relation between cytokines, LP, and the pathophysiology of left ventricular dysfunction during AVA, our findings support the existing data from the literature and underline the importance of programming a permanent pacemaker in such a way as to ensure the most physiological ventricular excitation sequence possible.
Authors' affiliations

M E Marketou, E N Simantirakis, D Nikitovic, S I Chrysostomakis, E A Zacharis, P E Vardas, Cardiology Department, University Hospital of Heraklion, Crete, Greece

Correspondence to: Professor Panos E Vardas, Cardiology Department, University Hospital of Heraklion, PO Box 1352, Stavrakia, Heraklion,

Crete, Greece; cardio@med.voc.gr

Accepted 13 September 2004

\section{REFERENCES}

1 Zile MR, Blaustein AS, Shimizu G, et al. Right ventricular pacing reduces the rate of left ventricular relaxation and filling. J Am Coll Cardiol 1987; 10:702-9.

2 Rosenqvist $M$, Bergfeldt $L$, Haga $Y$, et al. The effect of left ventricular activation sequence on cardiac performance during pacing. PACE 1996;19:1279-86.

3 Nielsen JC, Kristensen L, Andersen HR, et al. A randomized comparison of atrial and dual-chamber pacing in 177 consecutive patients with sick sinus syndrome. Echocardiographic and clinical outcome. J Am Coll Cardiol 2003;42:614-23.

4 Nielsen JC, Bottcher M, Nielsen $\Pi$, et al Regional myocardial blood flow in patients with sick sinus syndrome randomized to long-term single chamber or dual chamber pacing - effect of pacing mode and rate. J Am Coll Cardiol 2000;35: 1453-61.

5 Tsutsamoto T, Wada A, Matsumoto T, et al. Relationship between tumor necrosis factor-alpha production and oxidative stress in the failing hearts of patients with dilated cardiomyopathy. J Am Coll Cardiol 2001;37:2086-92. 